\title{
Uso do Modelo de Superfície de Resposta e Projeto Ótimo de Experimentos na Identificação de Danos Estruturais
}

\author{
Isabela Cristina da Silveira e Silva Rangel ${ }^{1}$ \\ Luciano dos Santos Rangel ${ }^{2}$ \\ Leonardo Tavares Stutz ${ }^{3}$
}

Instituto Politécnico do Estado do Rio de Janeiro, IPRJ, Universidade do Estado do Rio de Janeiro,

UERJ, Nova Friburgo, RJ

\begin{abstract}
Resumo. O presente trabalho aborda o problema de identificação de danos em uma viga de Euler-Bernoulli simplesmente apoiada utilizando as técnicas baseadas no domínio da frequência. Na formulação do problema de identificação de danos é utilizado o Modelo de Superfície de Reposta (MSR) em substituição de um Modelo de Elementos Finitos (MEF) da estrutura, com intuito de reduzir o custo computacional na solução do problema inverso. O MSR é determinado considerando os dados experimentais selecionados a partir do Projeto Ótimo de Experimentos, com objetivo de selecionar um conjunto ótimo de experimentos. Considerou-se o método Evolução Diferencial (ED) no problema em questão. Com os resultados obtidos, percebe-se que o Projeto Ótimo de Experimentos é de fundamental importância para a identificação de danos estruturais.
\end{abstract}

Palavras-chave. Modelo de Superfície de Resposta, Projeto Ótimo de Experimentos, Identificação de danos estruturais, Frequências naturais.

\section{Introdução}

Danos em estruturas de engenharia comprometem o desempenho e a integridade estrutural, resultando em perdas econômicas e ocasionando rupturas, colocando assim, vidas humanas em risco. A identificação de danos em um estágio inicial e o contínuo monitoramento da estrutura são questões de suma importância na engenharia, contribuindo para a redução dos custos de manutenção e de reparo, além de aumentar sua confiabilidade e sua vida útil.

Neste caso, estudos e ensaios devem ser realizados com intuito de fornecer recursos para uma correta avaliação da integridade da estrutura, podendo assim, estabelecer critérios da utilização da estrutura com segurança.

Os ensaios para a avaliação de danos estruturais podem ser ensaios destrutivos ou ensaios não destrutivos [3], dentre esses, destaca-se os ensaios não destrutivo, visto que,

\footnotetext{
${ }^{1}$ isabelacssilva@yahoo.com.br

${ }^{2}$ lucianosrangel@yahoo.com.br

${ }^{3}$ ltstutz@iprj.uerj.br
} 
são aqueles que não alteram a integridade da estrutura. Porém, estes ensaios são procedimentos locais, acarretando certas desvantagens, pois exigem um conhecimento a priori da região danificada, portanto, para contornar as desvantagens, métodos mais elaborados foram desenvolvidos, dentre eles, os métodos que estudam as mudanças nas características vibracionais da estrutura.

Os métodos de identificação de danos, baseados nas características vibracionais, geralmente são baseados em: dados modais (análise modal), dados no domínio do tempo e dados no domínio da frequência [2]. O presente trabalho considera parâmetros modais da estrutura, especificamente, as frequências naturais não amortecidas.

A literatura indica a utilização de técnicas de identificação de danos estruturais e monitoramento de estruturas fundamentadas no ajuste de um MEF. Mas, geralmente obtêm-se de um problema mal posto e um elevado custo computacional, inerente a essas técnicas. Para contornar esse aspecto, pode-se utilizar o MSR em substituição a um MEF da estrutura, onde este, apresenta como vantagem a redução do custo computacional para a solução de problemas inversos de identificação de danos [4].

Neste trabalho, a identificação de danos estruturais considera o ajuste de um MSR da estrutura, objetivando-se a minimização de uma função de erro definida a partir das frequências naturais experimentais e das correspondentes frequências previstas pelo MSR. O MSR é determinado considerando os dados experimentais selecionados a partir do Projeto Ótimo de Experimentos, com intuito de selecionar um conjunto ótimo de experimentos. E, a estrutura considerada no presente problema, trata-se uma viga de Euler-Bernoulli simplesmente apoiada, por fim, utiliza-se o método estocástico Evolução Diferencial (ED) para a resolução do problema inverso.

\section{Modelo de Superfície de Resposta}

O MSR é obtido através das relações entre parâmetros da estrutura e respostas de interesse. Desta forma, para uma dada resposta escalar y, tem-se

$$
y=f\left(\beta_{1}, \beta_{2}, \ldots, \beta_{n p}\right)+\varepsilon,
$$

onde $f\left(\beta_{1}, \beta_{2}, \ldots, \beta_{n p}\right)$ representa a relação entre a resposta e as variáveis independentes e $\varepsilon$ sendo o resíduo. Em geral, os parâmetros do modelo devem ser codificados, em $x_{i} \in[-1,1][7]$. Esta relação pode ser aproximada por polinômios de baixa ordem em algumas regiões relativamente pequenas do espaço definido pelas variáveis independentes, sendo então comumente utilizados modelos de primeira ou segunda ordem. Dessa forma, a Eq. (1) pode ser reescrita como

$$
\hat{y}=f\left(x_{1}, x_{2}, \ldots, x_{n p}\right)+\varepsilon=\hat{b}_{0}+\sum_{i=1}^{n p} \hat{b}_{i} x_{i}+\sum_{i=1}^{n p} \hat{b}_{i i} x_{i}^{2}+\sum_{i<j}^{n p} \sum_{j=2}^{n p} \hat{b}_{i j} x_{i} x_{j} .
$$

Para a determinação dos coeficientes da função de resposta, deve-se ter um número $n_{d}$ de dados maior, ou no mímino igual, ao número de coeficientes. Considerando o método dos mínimos quadrados, estima-se o vetor de coeficientes $\hat{\mathbf{b}}$ por

$$
\hat{\mathbf{b}}=\left(\mathbf{X}^{T} \mathbf{X}\right)^{-1} \mathbf{X}^{T} \mathbf{y}
$$


onde $\mathbf{y}$ é o vetor contendo resposta $y, \mathbf{X}$ é a denominada matriz de projeto, cujos componentes são obtidos dos parâmetros codificados $x_{i}, \hat{\mathbf{b}}$ o vetor contendo os coeficientes da função de resposta e $\varepsilon$ o vetor contendo os resíduos do modelo.

O MSR constitui-se de combinações de técnicas de Projeto de Experimentos e existem dois tipos principais de projeto: Projeto Composto Central (PCC) e Box-Behnken [6]. Classifica-se um PCC em três tipos: Circunscrito, Inscrito e Face Centrada, sendo estes diferenciados pela localização dos pontos axiais [7].

\subsection{Projeto Ótimo de Experimentos}

O Projeto Ótimo de Experimentos trata-se de uma metodologia onde seleciona um subconjunto particular de combinações que contenha informações suficientes sobre o domínio (conjunto de todas as combinações possíveis/candidatas do PCC). Para cada subconjunto ótimo, são selecionadas as combinações mediante o algoritmo de Federov [1], por fim, classifica-se o melhor subconjunto ótimo através do projeto D-ótimo.

O projeto D-ótimo busca maximizar o determinante da matriz de informação $\left(\mathbf{X}^{T} \mathbf{X}\right)$. Os elementos da diagonal principal representam a variância dos coeficientes do modelo $\left(b_{i j}\right.$, onde $\left.i=j\right)$ e os outros elementos $\left(b_{i j}\right.$, onde $\left.i \neq j\right)$ representam a covariância.

$$
\operatorname{det}\left(\mathbf{X}^{T} \mathbf{X}\right)=\frac{1}{\operatorname{det}\left(\mathbf{X}^{T} \mathbf{X}\right)^{-1}}
$$

No entanto, caso as matrizes não tenham a mesma quantidade de combinações, a comparação dos determinantes não pode ser realizada, devido à influência do número de combinações. Esta influência é eliminada, possibilitando a comparação das matrizes, por meio da matriz de momentos, dada por:

$$
\mathcal{M}=\left(\mathbf{X}^{T} \mathbf{X}\right) / n_{c}
$$

sendo $n_{c}$ o número de combinações. Considera-se $\mathbf{X}_{n_{1}}$ como melhor projeto em relação a $\mathbf{X}_{n_{2}}$, se $\operatorname{det} \mathcal{M}\left(\mathbf{X}_{n_{1}}\right)>\operatorname{det} \mathcal{M}\left(\mathbf{X}_{n_{2}}\right)$.

\section{Formulação do Problema Direto e Inverso}

Na estratégia de identificação de danos adotada, a integridade da estrutura é considerada como sendo continuamente descrita, no domínio do corpo, por um parâmetro estrutural denominado parâmetro nodal de coesão $(\beta)$ [9]. Este parâmetro está relacionado com a ligação entre os pontos materiais e pode ser interpretado como uma medida do estado de coesão local do material, onde $0 \leq \beta \leq 1$.

Neste trabalho, considerou-se que o dano afeta apenas as propriedades elásticas da estrutura, hipótese comumente adotada na literatura. Deste modo, a matriz de rigidez do MEF da estrutura pode ser escrita como

$$
\mathbf{K}\left(\beta_{h}\right)=\int_{\Omega} \beta(x) E_{0} I_{0} \mathbf{H}^{T}(x) \mathbf{H}(x) d \Omega,
$$


onde $\mathbf{H}$ é o operador diferencial discretizado, $E_{0}$ e $I_{0}$ são, respectivamente, os valores nominais do módulo de elasticidade e do momento de inércia de área e $\beta$ representa o campo de coesão no domínio elástico $\Omega$ da estrutura. A partir da Eq. (6), tem-se que a rigidez à flexão ao longo da viga é dada por

$$
E(x) I(x)=\beta(x) E_{0} I_{0} .
$$

Portanto, o parâmetro de coesão representa qualquer alteração, provocada pela presença de danos estruturais, na rigidez à flexão da estrutura. Por simplicidade, considerandose uma viga de seção transversal retangular e com módulo de elasticidade uniforme, o campo de coesão pode ser escrito como

$$
\beta(x)=\left(h(x) / h_{0}\right)^{3},
$$

onde $h_{0}$ e $h(x)$, indicam, respectivamente, a espessura nominal e a espessura da viga na posição $x$. No presente trabalho, as frequências naturais não-amortecidas serão utilizadas no problema de identificação de danos estruturais, sendo estas, obtidas a partir do seguinte problema de autovalor-autovetor generalizado, escrito na forma matricial como

$$
\mathbf{K} \Phi=\Lambda \mathrm{M} \Phi
$$

onde $\mathbf{M}$ é a matriz de massa e as matrizes modais $\boldsymbol{\Phi}$ e $\boldsymbol{\Lambda}$ constituem, respectivamente, a matriz de autovetores e autovalores, dadas por

$$
\boldsymbol{\Phi}=\left[\begin{array}{llll}
\boldsymbol{\Phi}_{1} & \boldsymbol{\Phi}_{2} & \ldots & \boldsymbol{\Phi}_{n}
\end{array}\right] \quad \boldsymbol{\Lambda}=\operatorname{diag}\left(\omega_{i}^{2}\right), \quad i=1, \ldots, n,
$$

onde $\boldsymbol{\Phi}_{i}$ é a i-ésima forma modal da estrutura e $\omega_{i}^{2}$ a frequência natural não-amortecida.

Para a formulação do problema inverso de identificação de danos no domínio da frequência, tem-se um funcional definido a partir da norma quadrática ponderada da diferença entre o vetor contendo as frequências naturais obtidas experimentalmente $\left(\mathbf{v}_{E}\right)$, o vetor contendo as frequências naturais previstas pelo $\operatorname{MSR}(\hat{\mathbf{v}}(\boldsymbol{\beta}))$,

$$
\min _{\boldsymbol{\beta}} \mathcal{F}=\min _{\boldsymbol{\beta}}\left(\left(\mathbf{v}_{E}-\hat{\mathbf{v}}(\boldsymbol{\beta})\right)^{T} \mathcal{W}\left(\mathbf{v}_{E}-\hat{\mathbf{v}}(\boldsymbol{\beta})\right)\right),
$$

sendo $\mathcal{W}$ uma matriz de ponderação. No presente trabalho, para a resolução do problema inverso, utilizou-se o algoritmo Evolução Diferencial [8].

\section{Resultados}

A estrutura considerada foi uma viga de aço simplesmente apoiada com 1,46 m de comprimento, $7,9375 \times 10^{-3} \mathrm{~m}$ de espessura, $7,62 \times 10^{-2} \mathrm{~m}$ de largura, momento de inércia de área $3,1756 \times 10^{-9} \mathrm{~m}^{4}$, módulo de elasticidade $2,07 \times 10^{11} \mathrm{~Pa}$ e massa específica $7,85 \times$ $10^{3} \mathrm{~kg} / \mathrm{m}^{3}$. A viga em questão foi discretizada pelo MEF em 24 elementos bidimensionais do tipo Euler-Bernoulli, onde cada elemento apresenta dois nós e cada nó possui dois graus de liberdade (GDL) e um parâmetro de coesão. Portanto, a estrutura possui 25 parâmetros nodais de coesão e, devido às condições de contorno, 48 GDL. 
No entanto, a partir de uma análise do comportamento da viga em relação às suas frequências naturais, observou-se que danos de mesma magnitude e em posições simétricas resultam nas mesmas variações nas frequências naturais [7]. Com isso, a viga foi considerada como sendo formada por subestruturas, diminuindo assim, o número de parâmetros nodais de coesão a serem utilizados na geração das superfícies de resposta.

As subestruturas são definidas através de parâmetros nodais de coesão simétricos.

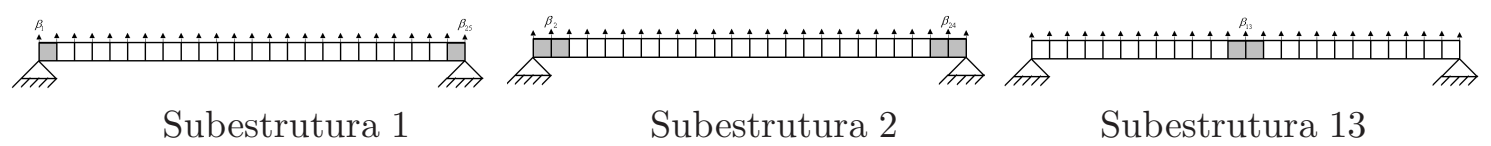

Figura 1: Divisão das subestruturas presentes na viga simplesmente apoiada.

Na identificação de danos utilizou o MSR das seis primeiras frequências naturais, sendo estas, superfícies do tipo quadrático com interação entre os parâmetros (QI) e para a obtenção do MSR foi adotado o PCC do tipo Circunscrito (CCC) [5]. Este projeto necessita de pontos fatoriais $\left(\beta_{-1}, \beta_{1}\right)$, pontos axiais $\left(\beta_{-\alpha}, \beta_{\alpha}\right)$ e ponto central $\left(\beta_{0}\right)$, sendo estes pontos, os limites das variáveis de entrada para a obtenção da superfície de resposta. Os limites considerados foram: $\beta_{-\alpha}=0,182, \beta_{-1}=0,512, \beta_{0}=0,729, \beta_{1}=1$ e $\beta_{\alpha}=1,331$.

O modelo QI possue 105 coeficientes, logo, necessita-se de um número de combinações maior ou igual ao número de coeficientes. Com isso, considerou projetos D-ótimos envolvendo 106, 107, 108, 150, 200, 500, 1000, 2000, 5000 e 8219 combinações, onde estas são escolhidas entre as 8219 combinações possíveis do CCC para as 13 subestruturas.

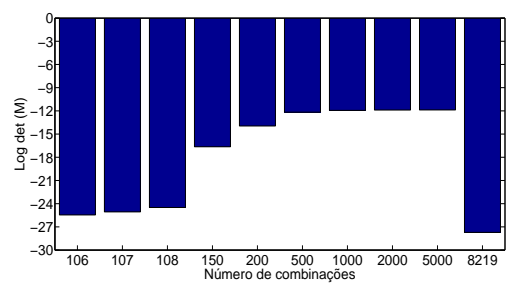

Figura 2: Logaritmo do determinante da matriz de momento.

O projeto D-ótimo com 200 combinações foi selecionado, onde este apresentou um elevado valor, para o determinante da matriz de momento e um número relativamente pequeno de combinações. A Tabela 1, apresenta os cenários de danos que serão considerados neste trabalho.

Tabela 1: Cenários de danos.

\begin{tabular}{|c|c|c|c|c|}
\hline Caso & Subestrutura & Posição $(\mathrm{m})$ & $h(x) / h_{0}$ & Nível de ruído $(\%)$ \\
\hline 1 & 5 & 0,2433 & 0,9 & 0 \\
\hline 2 & $5 ; 13$ & 0,$2433 ; 0,73$ & 0,$8 ; 0,8$ & 1 \\
\hline
\end{tabular}

As Figuras 3 e 4, apresentam a média das dez simulações utilizando o método ED para os dois cenários de danos e o erro relativo das seis primeiras frequências naturais, antes e depois da identificação de danos. 


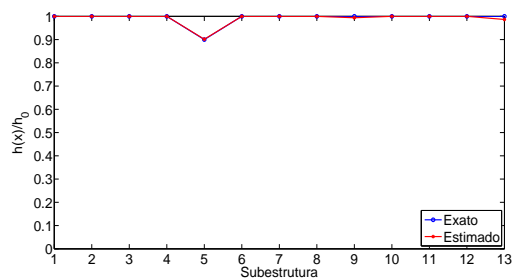

(a) Resultado da identificação de danos.

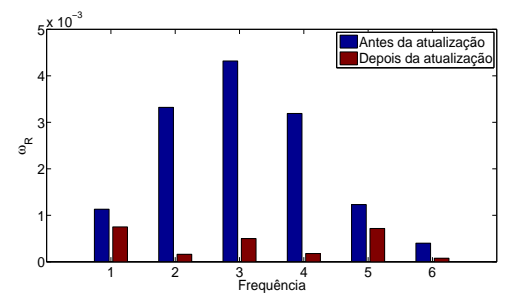

(b) Indicador de erro.

Figura 3: Identificação de danos para o Caso 1.

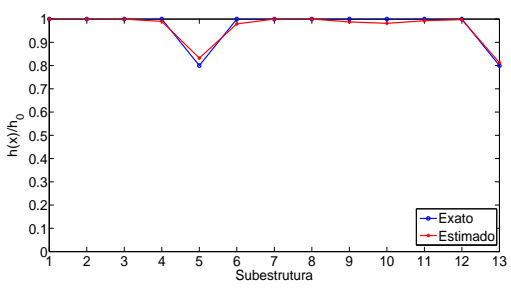

(a) Resultado da identificação de danos.

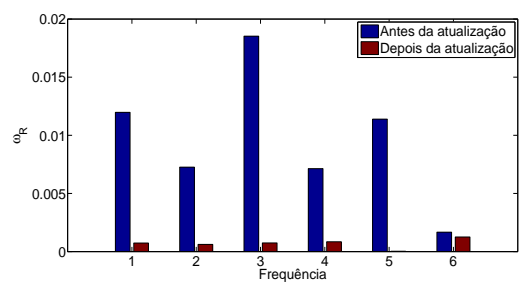

(b) Indicador de erro.

Figura 4: Identificação de danos para o Caso 2.

Nos resultados apresentados, é possível perceber que o MSR adotado localizou e quantificou o dano com boa acurácia e observa-se que os erros relativos das frequências naturais consideradas, diminuíram consideravelmente após a estimação dos parâmetros nodais de coesão, validando assim, o resultado. Em relação ao custo computacional, avaliou-se o tempo médio gasto no processo de identificação de danos tanto utilizando o MSR quanto o MEF - atualizando todos parâmetros da estrutura e atualizando as subestruturas.

Tabela 2: Custo Computacional.

\begin{tabular}{|c|c|}
\hline Modelo adotado & Tempo (s) \\
\hline MSR & 21 \\
\hline MEF - atualizando todos parâmetros da estrutura & 710 \\
\hline MEF - atualizando as subestruturas & 480 \\
\hline
\end{tabular}

A partir do resultado apresentado na Tabela 2, verifica-se que o MSR reduz consideravelmente o custo computacional no processo de identificação de danos. Por fim, podemos concluir que a abordagem proposta foi capaz de identificar com acurácia as subestruturas danificadas e a intensidade dos danos.

\section{Conclusões}

No presente trabalho, um MSR ajustado para as frequências naturais foi adotado para a identificação de danos estruturais em uma viga de Euler-Bernoulli simplesmente 
apoiada, considerando os dados experimentais selecionados a partir do Projeto Ótimo de Experimentos.

A partir dos resultados obtidos, observou-se que a estratégia foi capaz de localizar e quantificar as subestruturas danificadas com elevada acurácia, além de reduzir o custo computacional. Alguns aspectos podem ser levantados como sugestões para trabalhos futuros, dentre eles, a aplicação do MSR em estruturas do tipo viga, com outras condições de contorno, e estruturas do tipo placa.

\section{Agradecimentos}

Os autores agradecem ao IPRJ/UERJ pelo apoio concedido.

\section{Referências}

[1] P. F. Aguiar, et. al., Tutorial: D-optimal designs. Chemomrtrics and Intelligent Laboratory Systems. vol.30. pp. 199-210, (1995).

[2] R. A. P. Corrêa, Identificação de danos em estruturas bi-dimensionais via matriz de flexibilidade baseada em um modelo de dano contínuo. $129 \mathrm{f}$. Tese de Doutorado em Modelagem Computacional, Instituto Politécnico, Universidade do Estado do Rio de Janeiro, Nova Friburgo, (2013).

[3] L. F. Fadel Miguel, Identificação de sistemas e avaliação da integridade de estruturas treliçadas. Tese de Doutorado em Engenharia Civil, Universidade Federal do Rio Grande do Sul, Rio Grande do Sul, (2007).

[4] S. E. Fang e R. Perera, A response surface methodology based damage identification technique. J. Iop Poblishing, vol. 18, pp. 14,( 2009).

[5] Q. T. Guo, L. M. Zhang, Finite element model updating based on response surface methodology Proc. 22nd Int. Modal Analysis Conf. (Dearborn, MI), (2004).

[6] P. S. Lopes, Modelagem de problema inverso de detecção de danos por técnicas de identificação de parâmetros e de otimização. Tese de Doutorado em Engenharia Mecânica, Itajubá, pp. 135, (2010).

[7] I. C. S. S. Rangel, Identificação de danos estruturais a partir do modelo de superfície de resposta. 109 f. Dissertação de Mestrado em Modelagem Computacional, Instituto Politécnico, Universidade do Estado do Rio de Janeiro, Nova Friburgo, (2014).

[8] R. Storn, K. Price, Differential Evolution - A simple and efficient adaptive scheme for global optimization over continuous spaces. Journal of Global Optimization, vol. 11, pp. 341-359, Berkekey, (1997).

[9] L. T. Stutz, D. A. Castello, F. A. Rochinha, A flexibility-based continuum damage identification approach. Journal of Sound and Vibration, 279, pp. 641-667, (2005). 